\title{
Design of High Concentration Environmental Sensor Based on MEMS Chip
}

\author{
Buqu Zeng ${ }^{1}$ and Tao Kuang ${ }^{2}$ \\ ${ }^{1}$ Huanghuai University.Zhumadian,Henan.China.463000 \\ ${ }^{2}$ Xinxiang University.Henan China.453000 \\ Corresponding E-mail:zbqxxu@sohu.com
}

\begin{abstract}
There are increasingly urgent needs for low power, high performance, and low cost environmental sensor in environmental concentrator industry. A new type of MEMS environmental sensor based on MEMS thin film resistor and heat transduction is designed. Using clean room technology processing micron-size chip, help reduce post production costs. Preliminary experiment results demonstrate that the output voltage signal is proportional to the environmental concentration in normat working range, and the detection accuracy can reach less than $1 \%$.
\end{abstract}

Keywords: environmental sensors, MEMS, thin-fitm resistors, low power consumption, high environmental concentration

\section{Introduction}

With the development of social economy, more and more people attach importance to health care; home environmental concentrator-market is also growing. General household use of environmental equipment is to improve the use of molecular sieve environmental concentration, to relieve fatigue, to increase metabolism, and to improve human health functions. Only suitable conentration of environmental can achieve health function, and therefore the device needs to monitor the output environmental concentration. According to the national standard of environmental machine industry "YY0732-2009 medical environmental concentrators safety requirements" [1], when the environmental concentration is less than $50 \%$, the system need to lit red light alarm; when the environmental concentration is higher than $82 \%$, the system need to lit green light, marking system is working properly; and when the environmental concentration is between $50-82 \%$, che system need to lit yellow light. In the current detection method of gas concentration, there are many ways to detect the concentration of environmental, but the most commonly used electrochemical method is for low environmental concentration measurements. The sensor life expectancy is greatly shortened at high environmental concentrations, and is not widely applied in environmental concentrator.

\section{High Environmental Concentrations Sensor}

Currently, the main methods for high environmental concentration detection are zirconia environmental sensor and ultrasonic sensor. Zirconia environmental sensor is mainly based on the ionic conduction of stable zirconia at high temperature [2]. Detection method is that through the guide tube, the measured gas is introduced into the zirconia detection chamber, and then through heating element the zirconia is heated to operating temperature $\left(650^{\circ} \mathrm{C}\right.$ above), The concentration difference of environmental gas and the reference gas cause a potential difference, whereby the environmental concentration can be calculated. The advantage of this method is that is no influence of gas temperature and by using different guide tube, environmental content can be detected at various gas 
temperatures. This flexibility is being used in many industrial online testing. The disadvantage is slow reaction time, complex structure, and easily affected detection accuracy.

Ultrasonic measurement principle is that in a fluid filled pipe, when ultrasonic pulse passes along the fluid, in the downstream direction and the reverse flow direction has different propagation time. When gas flow rate is different, upstream and downstream time difference is different, thus gas flow rate can be detected by the time difference. The concentration of detection is also using the same ultrasonic pulse, under the dual gas composition; the concentration ratio of the two components is different, ultrasonic pulse propagation velocities in the gas are also different. Now environmental concentrator mainly use ultrasonic type environmental concentration sensor. This method is simple in principle, but the price is more expensive, and need more complex single sensor calibration work, so the cost is difficult to drop, limits its application in industry

\section{Environmental Detection Based on MEMS Chip}

Semiconductor processing technology is used in MEMS chip fabrication processing, with mass-production capability and high repeatability, thus the price is low and, the stability and economy of environmental sensors can be improved For measuring environmental concentration, thermal conductivity type en ironmental sensors is designed based on gas thermal conductivity differences. Ths study adopts the suspended thin film resistor for heating and temperature measurement. Suspended thin film resistor can effectively reduce the heat loss caused by heat conduction, and guarantee the independence between the resistors. Its workmg principle is shown in Figure 1.

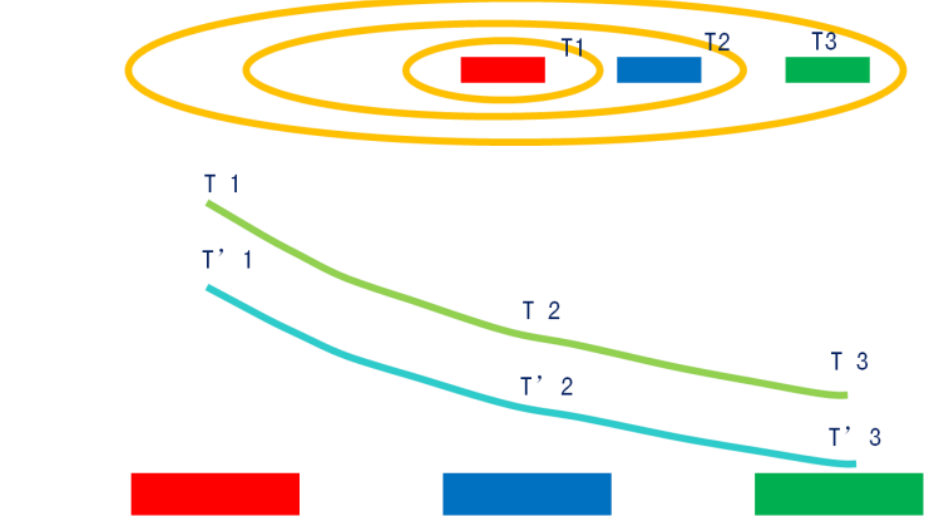

Figure 1. Detection Principle of Environmental Concentration Based on MEMS

In Figure 1, T1, T2 and T3 are suspended film platinum resistors, T1 works as the heater, emitting a constant heat to increase the flow air temperature. T2 and T3 work as temperature measurement devices. The measured gas flow into the gas chamber is heated o a high temperature in contact with the thermostat. When the gas flow, according to three basic ways of heat transfer: heat conduction, convection and radiation, the overall heat loss the overall heat loss as shown in equation one.

$Q=Q_{\text {convection }}+Q_{\text {conduction }}+Q_{\text {radiation }}$

Because of adopting suspended membrane resistor, its length to thickness ratio is 3000 or more, the heat conduction can be neglected [3]. At a constant temperature mode, sensor surface thermal radiation also changes very little, without consideration. Therefore, the heat loss of the sensor can be expressed as:

$Q_{\text {convection }}=\lambda m\left(T-T_{0}\right)$ 
Wherein, $\lambda$ is the thermal conductivity of the gas, $T$ is the sensed temperature, $T_{0}$ is the ambient temperature, and $\mathrm{m}$ is the mass flow rate of the gas. The thermodynamic theory shows that if the gas to be measured only binary gas mixture, the thermal conductivity of a mixed gas component to be measured gas concentration is linear, i.e., $\mathrm{C} 1=(\lambda-\lambda 0) /$ $(\lambda 1-\lambda 0)$, thermal conductivity of environmental and nitrogen is different, therefore, as long as the measured thermal conductivity of the mixed gas, wherein the environmental concentration can be known. The different environmental concentrations and flow rates of gas heat away different, by detecting the resistance value of T2 and T3 pre-calibrated values for comparison can be drawn information flow rate and environmental concentration of the gas. In order to ensure the accuracy, relatively independent platinum resistor is designed to detect the ambient temperature on the chip to be amended.

Then sensitivity is a key parameter of microbolometer which is defined as the output voltage divided by the incident radiant power (P. W. Kruse et al., 1997) can be expressed as Equation (2):

$S_{v}=\frac{\partial V_{o}}{\partial P}=\frac{\alpha \cdot \eta \cdot V_{f i d} \cdot T_{\mathrm{int}}}{\sqrt{G_{e f f}^{2}+\omega^{2} C^{2}} \cdot R_{s} \cdot C_{\mathrm{int}}}$

The thermal conductance is usually estimated by solving Fourier's first law of conduction and is approximate given by Equation (4) Error! Reference source not found.:

$G \approx 2 \sum_{i=1}^{n} \frac{k_{i} A_{i}}{l_{i}}$

Where, $n$ is the number of parallel layers forming $a \mathrm{~m}, k_{i}, A_{i}$, and $l_{i}$ are the thermal conductance, cross-sectional area, and length of thermal conductance of the $i^{\text {th }}$ layer of the region of the arms, respectively While the effective conductance of a microbolometer $G_{\text {eff }}$, affects the performance of the device (P. W. Krose et al., 1997) and is given by Equation (5).

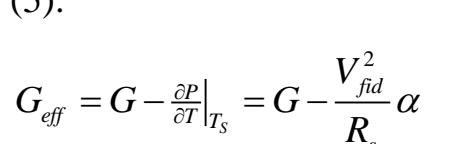

Where $V$ is a bias voltage $R_{s}$ is the resistance of the microbolometer, $\alpha$ is TCR of sensitive membrane. Then the effective TTC, $\tau_{\text {eff, }}$ can be solved with Equation (6):

The thermal capacitance $C$ of the microbolometer which can be expressed as Equation (6) (P. W. Kruse et. al (.) 1997):

$$
C=\sum_{i=1}^{n} V_{i} c_{i} \rho_{i}
$$

Where $n$ is number of parallel layers forming the microbolometer, $V_{i}, C_{i}$, and $\rho_{i}$ are the yolume, heat capacity, and density of the $i^{\text {th }}$ layer of each region of the microbolometer, respectively.

Another important parameter should be introduced before FEA.The effective TTC which is a measurement of the time required for the thermistor to respond to a change in the ambient temperature. The technical definition of TTC is the time required for a thermistor to change $63.2 \%$ of the total difference between its initial and final body temperature when subjected to a step function change in temperature, under zero power conditions(P. W. Kruse et al., 1997). It is also given by:

$\tau_{\text {eff }}=\frac{C}{G_{e f f}}$ 
To improve the simulation efficiency, the pulse voltage loading is equivalent DC voltages $V_{\text {eqbias }}$ as shown in Error! Reference source not found. (c), which is equivalent to the pulse voltage bias in energy: $V_{\text {eqbias }}$ can be solved from the use of Equation (8):

$\frac{V_{\text {eqbias }}^{2}}{R_{e}} \times T_{\text {star }}=\frac{V_{\text {fid }}^{2}}{R_{e}} \times T_{\mathrm{int}}$

The rule-of-thumb for TTC and frame rate of the detector is given by Equation (9) (Seniz E Kucuk et. al., 2011) :

$$
\tau \leq \frac{1}{2 \times \text { frame rate }}
$$

Where, the frame rate is commonly given as $60 \mathrm{fps}$ for high speed applications. Therefore, the time constant should not exceed $8.3 \mathrm{~ms}$. Considering that the TTC of the model used here is $5.4 \mathrm{~ms}$, it would function acceptably at a frame rate of $60 \mathrm{~Hz}$.

\section{MEMS Chip and Testing}

MEMS chip processing flow chart used in this study as shown in Flgue 2. By (100) type silicon (a), first deposited multilayer films as-a supporting structure (b), then by stripping fabrication resistance wire (c), followed by dry etching to open etching window (d), and then release the film by a wet etching process (e), and finally produced film resistor wire (f). From the point of manufacturing process, Sensor processing process is simple and controllable, can guarantee ahigher yield $(4,5)$.

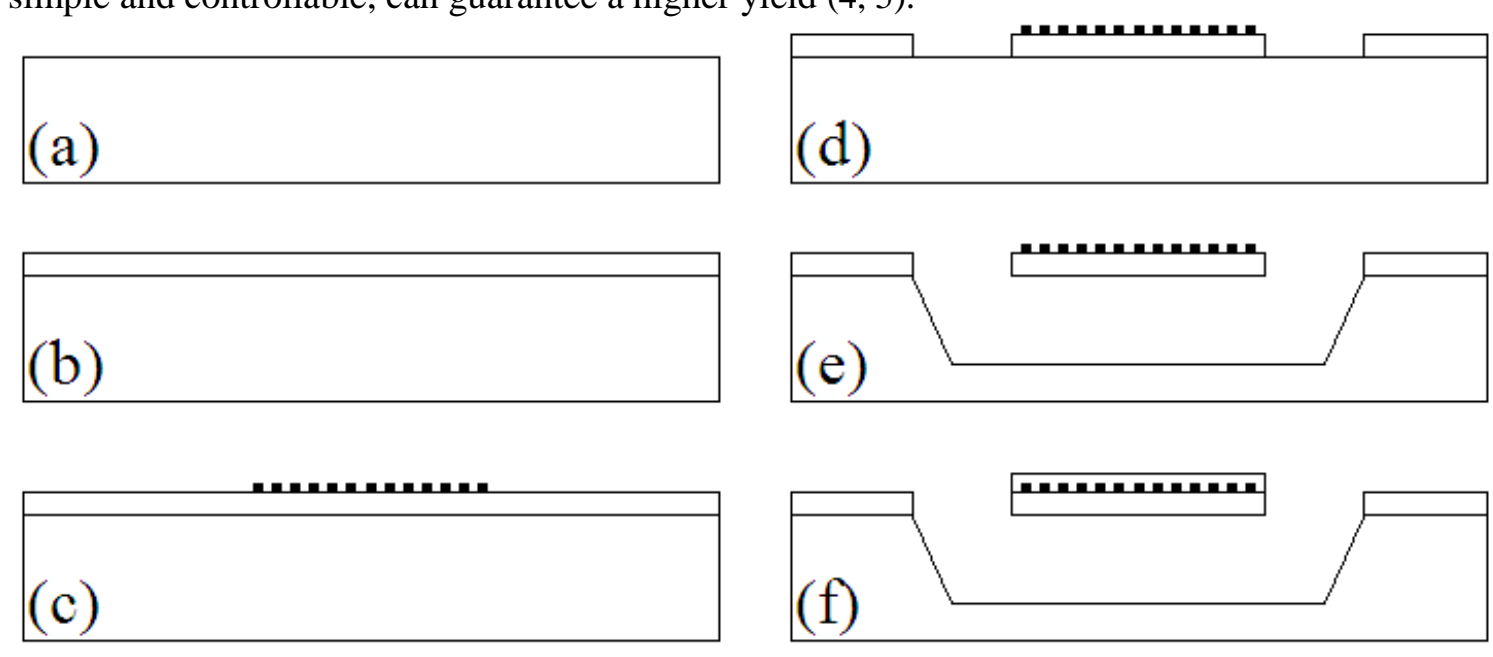

Figure 2 Eabrication Process of Thermal Conductivity Type Environmental Sensor

a) substrate silicon; (b) sedimentary supporting membrane; (c) production metal electrode; (d) etching corrosion window; (e) etching silicon substrate; (f) the sedimentary protective film. 


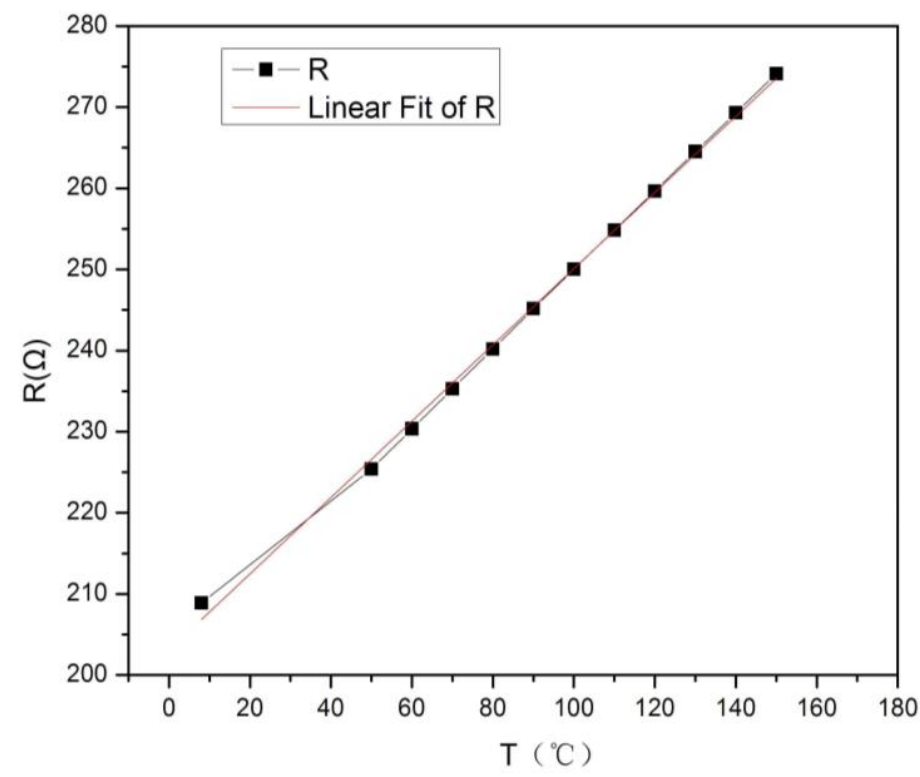

Figure 3. Relation Curve between Resistance and Temperature of Thin Film Pt Resistor on Chip

For the finished chip, we first calibrated the temperature coefficient of resistance of the actual device. Thin film platinum resistance versus temperature curve is shown in Figure 3 , according to the measured resistance value and calculation, the thin film platinum resistance temperature coefficient of resistance is $0.0024 /{ }^{\circ} \mathrm{C}$. The completely packaged chips are tested using different ratios of environmental and nitrogen gas mixture, with a constant current control circuit The results are shown in Figure 4. We can see that the environmental concentration and voltage has a good linear relationship. And when the environmental concentration changed $1 \%$, the output voltage changed at $\mathrm{mV}$ level, and the circuit detects the signal up to the Microvolt level, therefore, the actual detection accuracy up to $1 \%$ or less can be achieved.

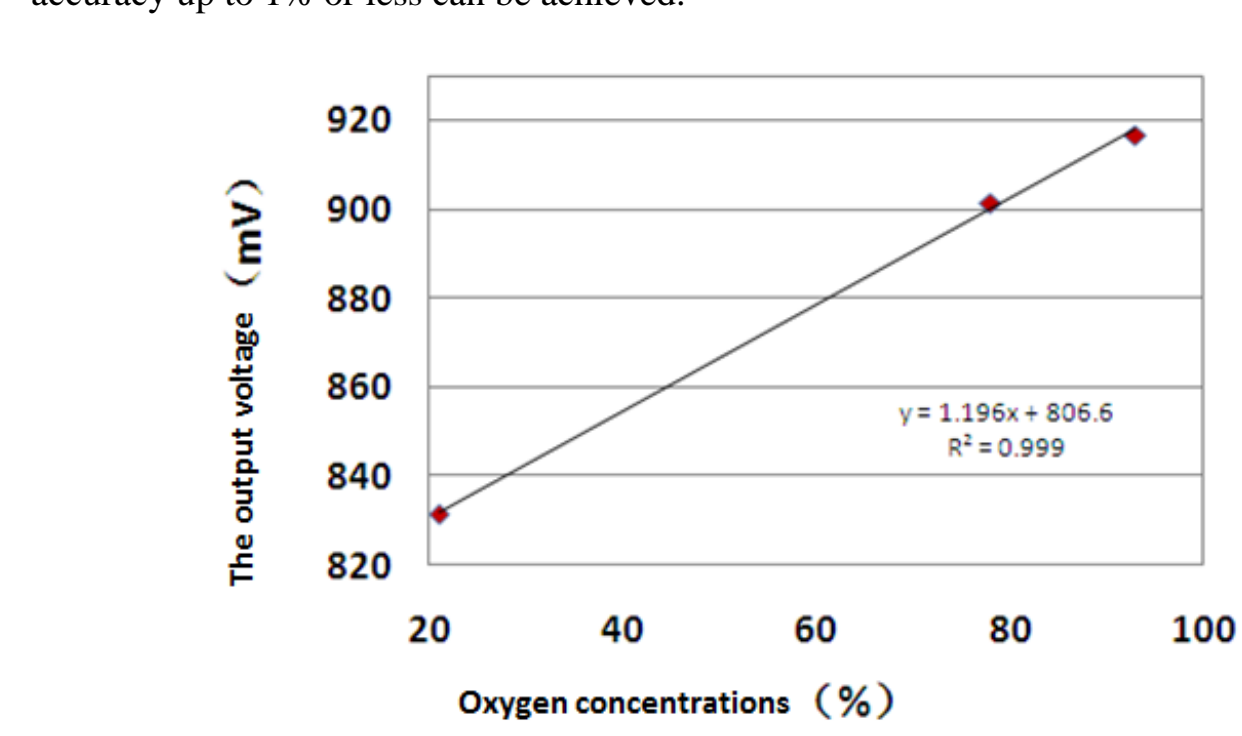

Figure 4. Relation Curve between Environmental Concentration and Output Voltage

Indoor Environment Monitoring Network System Introduction 
The traditional library environment mostly manually collected data, including digital hygrometer, illumination instrument. Abroad as early as the 1970s began to study the temperature and humidity control technology, which uses a combination of analog instrumentation, acquisition of temperature and humidity information and instructions, recording and control, but the accuracy and consistency of data is not guaranteed. Based on RS485 cable detection techniques require a large amount of laying transmission lines and equipment for centralized power in the economy and flexibility, there are some problems [4]. Existing techniques to monitor the indoor environment is to be automated, unmanned direction, and wireless sensor networks (Wireless Sensor Network) are equipped with these features. Wireless sensor networks are deployed in the area to monitor a large number of cheap micro-sensor nodes through wireless communication to form a multi-hop ad hoc networks [5]. Common wireless communication protocol specifications are summarized in Table 1.

ZigBee technology is a wireless transmission technology agreement with a close low complexity, low power, low-rate, short-latency, high network capacity, low cost, reliable, safety features, mainly used for short distance, data transfer between low-power and the transmission rate is not high, and a variety of electronic dexices typically have periodic data, intermittent data and low latency data transfer applieations [6]. Therefore, the use of the characteristics of ZigBee technology, combined with the special needs of the University Library environmental monitoring, offered to ZigBee + GPRS technology, monitoring programs, both from the hardware and sof ware design completed university library environment monitoring system.

Table 1. The Technical Specifications of Several Wireless Communication

\begin{tabular}{|c|c|c|c|c|}
\hline & Bluetooth & ZigBee & $W_{1-F i}$ & Infrared \\
\hline Range & $10 \mathrm{~m}$ & $100 \mathrm{~m}$ & $1 \mathrm{~m}$ & $75 \mathrm{~m}$ \\
\hline Rate & $1 \mathrm{Mbps}$ & $11-600 \mathrm{Mbps}$ & $3 \mathrm{Mbps}$ & 2503Mbps \\
\hline Band & $2.4 \mathrm{GHz}$ & $2.4 \mathrm{~Hz}, 5 \mathrm{MHz}$ & 980nm & $869 / 915 \mathrm{MHz} \quad 2.4 \mathrm{GHz}$ \\
\hline Standard & 802.15 .1 & 80211 & Not uniform & 802.15 .4 \\
\hline Power & High & Higher & Lower & Low \\
\hline Costs & General & High & Low & Low \\
\hline
\end{tabular}

\section{Monitoring System Design}

Academic Library Environment Monitoring System main function is to achieve long-term and efficient on-line monitoring and transport-related environmental parameters. Making the overall program design, first needs to consider the environmental characteristics of the University Library, monitoring point layout and construction of the network system and other factors to determine monitoring parameters, hardware selection and transport protocol settings, etc., combined with various hardware modules and transmission protocols the set design software monitoring section.

\section{Monitoring System Hardware Design}

Monitoring system hardware design includes composition and network coordinator node monitoring sites designed. The functional requirements of the system, the architecture shown in Figure 5. 


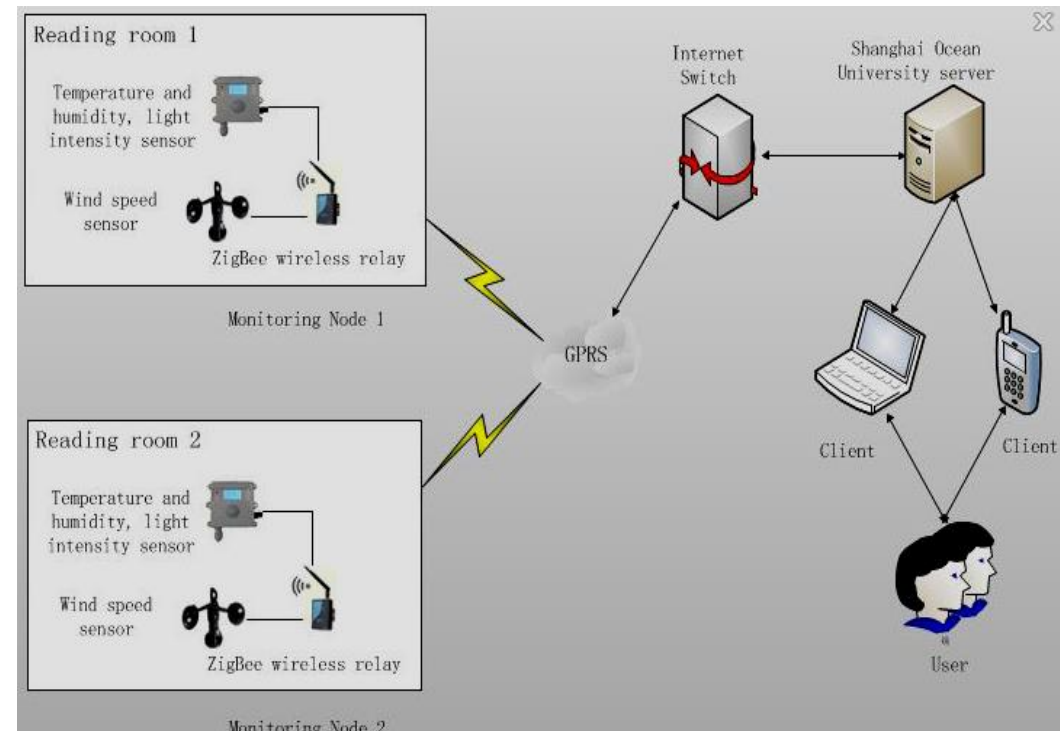

Figure 5. The Structure of Library Environment Monitoring System

\section{Library Based on ZigBee Technology Monitoring System}

Shown in Figure 6, this experiment chose Shanghai Ocean University Information Center on the first floor and second floor reading room, because the light intensity is stronger, larger space, the number of frequent aceess, is not conducive to wiring, requiring the use of wireless communication protocols transmission signal. The nodes in the network are arranged in a suitable doeation in the reading room, all nodes are hung module temperature and humidity light intensity, such as sensors, near the sunny side of the window to form a numerical comparison atso hung sensor. 1min collected once every terminal node data, the length of lime interval can also be prepared in accordance with the needs of the administrator as shown in F gure 4 .
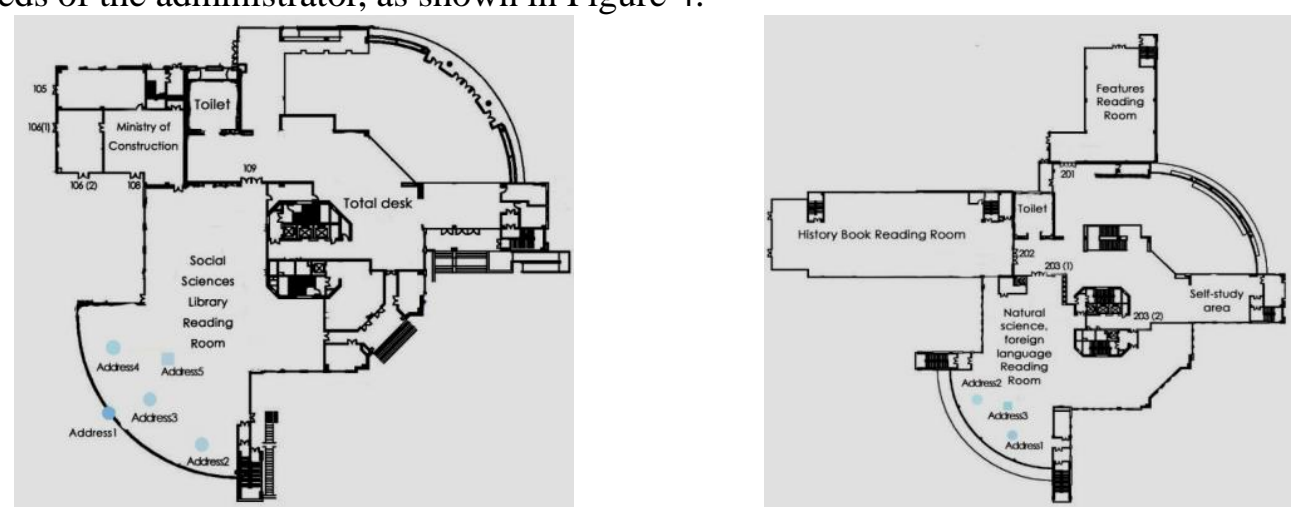

Figure 6. Plane Graph of the Library Reading Room

At the same time, through the ZigBee wireless sensor network and GPRS technologies, when collecting data once over the threshold, the system will automatically alarm. ZigBee wireless sensor networks can also be combined with automatic control technology, depending on the circumstances, the automatic control system to adjust the temperature of the air conditioning and curtains opening and closing, etc., to improve the efficiency of the staff of the university library, the ambient temperature to maintain optimal library.Shown in Figure 7. 


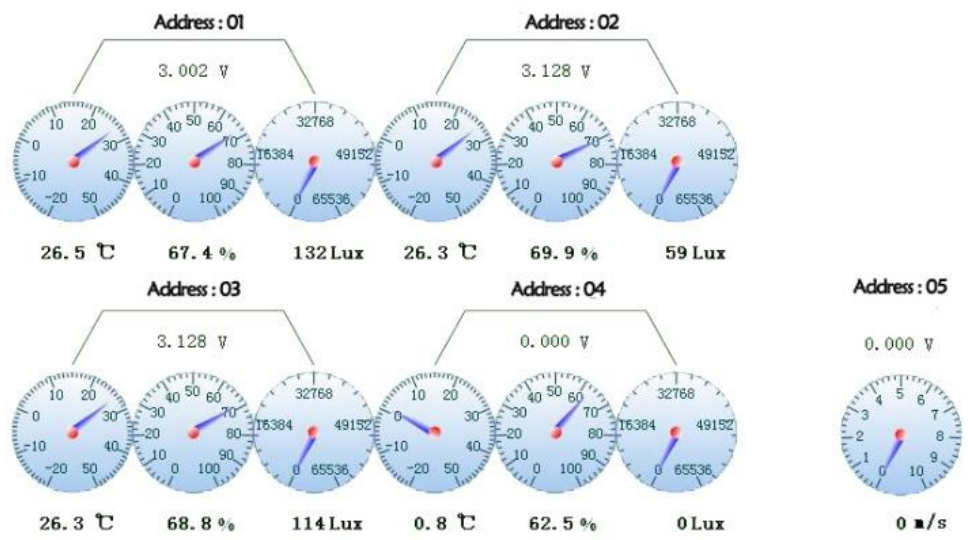

Figure 7. Interface of the Library Real-Time Monitoring Platform

\section{Conclusion}

Environmental sensor in automotive electronics, medical electronics and other industries has a wide range of applications. From the envîronmental sensor environmental as a starting point, MEMS chip is designed based on the thermal conductivity of the environmental sensor principle. The finished chip was verified through preliminary experiments, the output signal is proportional to the concentration of environmental, detecting environmental concentration of about $1 \%$ accuracy Using this chip can reduce the system cost and power consumption of environmental sensors, increasing the efficiency and service life, meet the needs of accurate monitoring used in the medical environmental concentrator and can be extended to bther âpplications and industries.

\section{References}

[1] Environmental industry nationar standard, "YY0732-2009 medical environmental concentrators' safety requirements".

[2] T. Feng, "Development zireonia substrate type NOx gas sensor", the sensor in the world, vol. 6, (2014).

[3] B, Du, "Thermal conductivity gas sensor principle and work to improve the detection", chemical engineering and equipment, vol. 2 (2010).

[4] L. Xu, Z. Dai and T. Li, "IC Compatible Fusion of Micromachined Hotplatform and Nanostructured Porous Film for High-Performance Gas Sensors", Transducers 2013, Barcelona, Spain, (2013) June $16-20$.

[5] L. Xu, T. Li and R. Zheng A Low Power Catalytic Combustion Gas Sensor Based on a Suspended Membrane Microhotplate" IEEE NEMS 2011, Kaohsiung, Taiwan, (2011) February 20-23.

[6] Z. Liu, L. Liu and W, Li, "Research greenhouse environment monitoring system based on ZigBee networks", Agricultural Mechanization Research, (In Chinese), vol. 10, (2014), pp. 218-222.

[7] L. Sun, J. Li and Y. Chen, "wireless sensor networks", Beijing: Tsinghua University Press, (In Chinese), (2005) pp. 6-7.

[8] D. M. Han and J. H. Lim, "Design and Implementation of Smart Home Energy Management Systems based on ZigBee", IEEE Transaction on Consumer Electronics, vol. 56, no. 3, (2010), pp. 1417-1424.

[9] R Gu, Y. Tian and E. Ekici, "Real-time multimedia processing in video sensor networks", Image Commun., vol. 22, no. 3, (2007), pp. 237-251.

\section{Author}

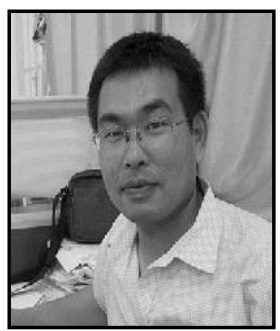

Buqu Zeng, assisting professor, Research direction: SCM. 